\title{
A prospective study on quality in endoscopic retrograde cholangiopancreatography (ERCP): trend in Italy from the REQUEST study
}

Authors

Giulio Donato ${ }^{1}$, Pietro Occhipinti ${ }^{1}$, Loredana Correale ${ }^{1}$, Marco Spadaccini ${ }^{2,3}$, Alessandro Repici ${ }^{2,3}$, Andrea Anderloni ${ }^{2}$, Alessandro Fugazza ${ }^{2}$, Piergiorgio Mosca ${ }^{4}$, Andrea Tringali ${ }^{5,6}$, Guido Costamagna ${ }^{5,6}$, Milutin Bulajic ${ }^{7,8}$, Giovanni de Pretis $^{9}$, Armando Gabbrielli ${ }^{10}$, Francesco Maria Di Matteo ${ }^{11}$, Roberto Faggiani ${ }^{12}$, Mohammad Ayoubi ${ }^{13}$, Luca De Luca $^{14}$, Paolo Cantù ${ }^{15}$, Matteo Blois ${ }^{16}$, Iginio Dell’Amico ${ }^{17}$, Attilio Maurano ${ }^{18}$, Maria Flavia Savarese ${ }^{19}$, Giampiero Manes $^{20}$, Raffaella Ferraro ${ }^{21}$, Mauro Barberis ${ }^{22}$, Cesare Hassan ${ }^{23}$, The REQUEST Group

\section{Institutions}

1 Gastroenterology Unit, Department of Oncological and Specialty Medicine, Ospedale “Maggiore della Carità”, Novara, Italy

2 Digestive Endoscopy Unit, Division of Gastroenterology, Humanitas Clinical and Research Hospital-IRCCS, Rozzano (MI), Italy

3 Digestive Endoscopy Unit, Division of Gastroenterology, Humanitas Clinical and Research Hospital-IRCCS, Rozzano (MI), Italy; Department of Biomedical Sciences, Pieve Emanuele, Milano, Italy

4 Division of Gastroenterology, Department of Gastroenterology \& Transplantation, AOU Ospedali Riuniti, Ancona, Italy

5 Digestive Endoscopy Unit, Fondazione Policlinico Universitario A. Gemelli IRCCS, Roma, Italy

6 Centre for Endoscopic Research Therapeutics and Training (CERTT), Università Cattolica del Sacro Cuore, Roma, Italy

7 Gastroenterology and GI Endoscopy Unit, University Hospital of Udine, Udine, Italy

8 Mater Olbia Hospital-Qatar Foundation Endowment \& Gemelli Foundation, Gastroenterology and GI Endoscopy Unit, Olbia (OT), Italy

9 Gastroenterology and Digestive Endoscopy Unit, Ospedale S. Chiara, Trento, Italy

10 Digestive Endoscopy Unit, AOUI Verona, Italy

11 Operative Digestive Endoscopy Unit, Policlinico Universitario Campus Bio-Medico di Roma, Roma

12 Gastroenterology and Endoscopy Unit, Department of Oncological and Specialty Medicine, S. Camillo Forlanini Hospital, Rome, Italy

13 Gastroenterology and Endoscopy Unit, GradenigoHumanitas, Torino, Italy

14 Gastroenterology and Digestive Endoscopy Unit, A.O. Ospedale Riuniti Marche Nord, Pesaro, Italy

15 Gastroenterology and Endoscopy Unit, Fondazione IRCCS Ca' Granda Ospedale Maggiore Policlinico, Department of Pathophysiology and Transplantation, Università degli Studi di Milano, Milan, Italy
16 Interventional Endoscopy Unit, Azienda USL Toscana Nord-Ovest, Livorno, Italy

17 Endoscopy Unit, Apuane Hospital, Massa-Carrara, Italy

18 Operative Endoscopy Unit, Azienda Ospedaliera Universitaria di Salerno, Italy

19 Gastroenterology and Gastrointestinal Endoscopy, General Hospital, Sanremo (IM), Italy

20 Department of Gastroenterology and Digestive Endoscopy, ASST Rhodense Garbagnate Milanese, Milano, Italy

21 Gastroenterology and Digestive Endoscopy Unit, Ospedale S. Andrea, ASL VC, Vercelli, Italy

22 Gastroenterology Unit, Policlinico di Monza, Monza (MB), Italy

23 Department of Gastroenterology, Nuovo Regina Margherita Hospital, Roma, Italy

24 Pancreatic/Biliary Endoscopy \& Endosonography Division, Pancreas Translational \& Clinical Research Center, San Raffaele Scientific Institute, Vita Salute San Raffaele University, Milan, Italy

25 IRCCS - ISMETT, Endoscopy Service, Department of Diagnostic and Therapeutic Services, Palermo, Italy

26 Digestive and Operative Endoscopy Unit, Niguarda-Ca Granda Hospital, Milan, Italy

submitted 18.3.2021

accepted after revision 7.6 .2021

Bibliography

Endosc Int Open 2021; 09: E1563-E1571

DOI 10.1055/a-1531-4691

ISSN 2364-3722

(C) 2021. The Author(s).

This is an open access article published by Thieme under the terms of the Creative Commons Attribution-NonDerivative-NonCommercial License, permitting copying and reproduction so long as the original work is given appropriate credit. Contents may not be used for commercial purposes, or adapted, remixed, transformed or built upon. (https://creativecommons.org/licenses/by-nc-nd/4.0/)

Georg Thieme Verlag KG, Rüdigerstraße 14,

70469 Stuttgart, Germany 
Corresponding author

Giulio Donato, MD, Gastroenterology Unit, Department of

Oncological and Specialty Medicine, AOU "Maggiore della

Carità”, Corso Mazzini 18, 28100, Novara, Italy

Fax: +3903213733345

giulio.donato@maggioreosp.novara.it

Supplementary material is available under https://doi.org/10.1055/a-1531-4691

\section{ABSTRACT}

Background and study aims Endoscopic retrograde cholangiopancreatography (ERCP) is a complex procedure with a relatively high rate of adverse events. Data on training of operators and fulfillment of quality indicators in Italy are scarce. The goal of this study was to assess the overall quality of ERCP in Italy compared to international standards.

Patients and methods This was a prospective, observational study from different Italian centers performing
ERCP. Operators answered a questionnaire, then recorded data on ERCPs over a 1-to 3-month period.

Results Nineteen Italian centers participated in the study. The most common concern of operators about training was the lack of structured programs. Seven/19 centers routinely used conscious sedation for ERCP. Forty-one experienced operators and 21 trainees performed 766 ERCPs: a successful deep biliary cannulation in native-papilla patients was achieved in $95.1 \%$ of cases; the post-ERCP pancreatitis (PEP) rate was $5.4 \%$ in native-papilla patients; cholangitis rate was $1.0 \%$; bleeding and perforation occurred in $2.7 \%$ and $0.4 \%$ of the patients, respectively.

Conclusions This study revealed that, overall, ERCP is performed in the participating Italian centers meeting good quality standards, but structured training and sedation practice are still subpar. The bleeding and perforation rate slightly exceeded the American Society of Gastrointestinal Endoscopy indicator targets but they are comparable to the reported rates from other international surveys.

\section{Introduction}

Endoscopic retrograde cholangiopancreatography (ERCP) has evolved into a predominantly therapeutic endoscopic-radiologic procedure. In spite of the advance in technology and devices, ERCP is still one of the most technically demanding endoscopic procedures, with relatively high adverse event $(A E)$ rates, ranging from $3 \%$ to $15 \%$ [1-5]. Appropriate training, experience, and competence are required when performing such a complex endoscopic procedure. In recent years, healthcare authorities have shown increasing interest in improving and standardizing endoscopy quality. Accordingly, in 2015 the American Society for Gastrointestinal Endoscopy (ASGE) provided an updated list of quality indicators for ERCP $[6,7]$; similarly, the European Society for Gastrointestinal Endoscopy (ESGE) recently published a set of performance measures for pancreato-biliary endoscopy [8]. Nevertheless, data on real-life fulfillment of these indicators are still scarce [9-14].

The REQUEST study (REte sulla QUalità della ERCP, dei Servizi di endoscopia e del Training - the Italian for "Network on ERCP, endoscopy services and training quality") is the first multicenter prospective study conducted in Italy with the goal to collect data on ERCP performance in a nationwide setting.

The aim of this study was to assess the overall quality of ERCP in Italy, compared to international standards. We collected data from facilities with different geographic locations, different volume of procedures as well as different levels of expertise. The quality of ERCP training programs was also investigated.

\section{Patients and methods}

In September 2016, a board of eight recognized Italian experts in ERCP designed this prospective, observational study, which was presented to centers performing ERCP at an Italian meeting on interventional endoscopy. Seventy-five centers were then contacted by the board to participate in the study.

Baseline characteristics of endoscopists and centers were collected at study entry. The participating centers were asked to answer a questionnaire about their facility details (No. of beds, availability of radiological services and endoscopic ultrasound, etc.), organization (No. of endoscopists, informed consent, ERCP setting, sedation practice, etc.) and operator characteristics (education, training time, no. of procedures performed in their career and per year, etc.).

Then, each center prospectively recorded patient and procedural data related to any consecutive ERCP procedure on a digital spreadsheet. A wide range of pre-, intra- and post-procedural parameters (i. e., patient demographics and clinical characteristics, indications, ERCP procedure details, accessories, efficacy and safety outcomes) were included. The file was compiled over a time frame of 1 to 3 months. This short time interval was chosen to allow the most accurate registration of the numerous parameters requested and to avoid drop-outs and improve adherence in compilation.

The primary endpoints were the rates of native papilla cannulation, successful stone extraction, successful stent placement for distal obstruction, and AEs. As secondary outcomes, regression analyses were performed in order to identify any possible association between patient- or operator-related characteristics and all primary outcomes. 
The study protocol was approved by the institutional review board at each participating center and it conformed to the ethical guidelines of the Declaration of Helsinki. Patients were enrolled after a written informed consent was obtained.

\section{Definitions}

The centers were considered as low- (<100 ERCPs), medium(100 to 300 ERCPs) and high- (>300 ERCPs) volume basing on the reported number of ERCPs performed in the preceding year (2015), similarly to previously implemented cut-offs [15, $16]$.

ERCP endoscopists were defined according to their competence as experienced - those who performed ERCP independently - and trainees - those who were training in ERCP.

ERCP difficulty was graded according to the modified Schutz classification [17].

Post-ERCP AEs were defined and graded according to the classification proposed by Cotton et al. [18].

\section{Statistical analysis}

Data analysis

Analyses were performed using the $R$ software package version 3.5.1 (2018-07-02) [R Core Team - A Language and Environment for Statistical Computing R Foundation for Statistical Computing Vienna, Austria 2016]. Descriptive statistics were used to characterize the study data. Continuous data were presented as means with standard deviations, whereas categorical data were presented as proportions. The potential factors associated with primary endpoints were assessed using a univariable analysis. The univariable analysis was conducted using the chi-squared test for categorical variables and the Student $t$ test for continuous variables. We also performed univariable and multivariable logistic regression analyses for the associations between study variables and the occurrence of complications, as described in detail below.

An initial univariable logistic regression analysis was performed to identify possible explanatory variables associated with the endpoint "post-ERCP AE". Variables of potential significance $(P<0.10)$ were entered into a multivariate model. Independent risk factors were expressed as the odds ratio (OR) with $95 \%$ confidence interval $(\mathrm{Cl})$. Statistical significance was set at $P<0.05$. In our study, there was a possible source of nonindependence of data, being the patients treated in different centers (clustered). Therefore, an adjustment by using clustered standard error was required for this potential environmental bias in estimating the parameters of regression. We also tested the independent factors for multicollinearity to detect critical correlations among the independent factors, as this may introduce problems in the estimation of the model coefficients. For this purpose, we employed the variance inflation factor (VIF) as a diagnostic test. This is because a VIF greater than 2.0 indicates a critical level of multicollinearity.

\section{Results}

From September 2016 to March 2018, 19/75 (25\%) centers, mostly located in Central and Northern Italy, joined this prospective, observational study after obtaining approval from the local institutional review board. The study centers were located as follows: 11 (57.9\%) in the North, 7 (36.8\%) in the center and $1(5.3 \%)$ in the South of Italy. The majority of the centers $(11 / 19,57.9 \%)$ were non-academic. Questionnaires detailing usual practice and experience were filled out by 62 ERCP endoscopists (ERCPists). Case record forms on patient characteristics, procedures, AEs, and clinical outcomes were received for 766 procedures.

\section{Center characteristics and facilities}

High-volume centers were 9/19 (47\%), while medium-volume centers were 10/19 (53\%). No low-volume center participated in the study. D Table 1 summarizes the characteristics of the study centers. Endoscopic ultrasound (EUS) was only available in 12 of 19 centers (63\%). An interventional radiology service for percutaneous transhepatic cholangiography (PTC) was available in 17 of 19 centers (89\%). Two centers (11\%) were located in facilities that offered neither EUS nor PTC services.

\section{Medical/nurse staffing}

On average, each center had 3.3 ERCPists (range, 1-5). Experienced operators were one to five per center (mean, 2.16; total 41 ), corresponding to $68 \%$ (range, 33-100\%) of all the ERCPists participating in the study. Trainees were one to four per center (1.21 on average), with an equal distribution between academic and non-academic centers. Overall, 15 of 19 centers (78.9\%) had nurses dedicated to ERCP procedures.

\section{Sedation practice}

Seven of 19 centers (37\%) routinely used conscious sedation (midazolam \pm pethidine), while eight of 19 (42\%) provided deep sedation with propofol and the remaining 4/19 (21\%) used general anesthesia ( $\triangleright$ Fig. 1). High-and medium-volume centers showed a nominal difference regarding the attendance of an anesthesiologist to the ERCP procedures (7 of 9-67\% - vs 5 of $10-50 \%$ - respectively; $P=0.630$ ).

\section{Prevention of post-ERCP pancreatitis}

Fourteen of 19 centers (73.6\%) used a prophylaxis based on non-steroidal anti-inflammatory drugs (NSAIDs) for post-ERCP prophylaxis (PEP) in all procedures, four of 19 centers $(21.1 \%)$ administered PEP prophylaxis only in high-risk patients and 1/ 19 (5.3\%) did not use PEP prophylaxis in any circumstances.

\section{Antibiotic prophylaxis}

The majority of the centers (17 of $19,89.5 \%$ ) used antibiotic prophylaxis for selected patients or procedures, following published guidelines [8]. 
- Table 1 Characteristics of the centers and profile of the operators. ${ }^{1}$

\begin{tabular}{|c|c|c|c|}
\hline Variable & $\begin{array}{l}\text { Overall } \\
(n=19)\end{array}$ & $\begin{array}{l}\text { High volume } \\
(n=9)\end{array}$ & $\begin{array}{l}\text { Medium volume } \\
(n=10)\end{array}$ \\
\hline \multicolumn{4}{|l|}{ Hospital size } \\
\hline Large ( $\geq 600$ beds), n (\%) & $9(47)$ & $6(67)$ & $3(30)$ \\
\hline Medium (200 to 599), n (\%) & $8(42)$ & $2(22)$ & $6(60)$ \\
\hline Small (<200), n (\%) & $2(11)$ & $1(11)$ & $1(10)$ \\
\hline \multicolumn{4}{|l|}{ Hospital setting of ERCP } \\
\hline Gastroenterology unit with beds & $11(58)$ & $5(56)$ & $6(60)$ \\
\hline Independent endoscopy service & $5(26)$ & $3(39)$ & $2(20)$ \\
\hline Endoscopy service part of a surgical unit & $3(16)$ & $1(11)$ & $2(20)$ \\
\hline \multicolumn{4}{|l|}{ ERCP time organization } \\
\hline ERCP performed $24 / 7$ & $8(42)$ & $5(56)$ & $2(20)$ \\
\hline ERCP performed 5 days a week (only in the morning) & $8(42)$ & $3(33)$ & $5(50)$ \\
\hline ERCP performed on specific days of the week & $2(11)$ & $1(11)$ & $1(10)$ \\
\hline ERCP performed only in the presence of trained operators & $1(5)$ & $0(0)$ & $1(10)$ \\
\hline \multicolumn{4}{|l|}{ Medical staffing } \\
\hline No. of employed endoscopists (mean, range) & $8(3-19)$ & $8.6(3-19)$ & $7.7(3-13)$ \\
\hline No. of endoscopists performing ERCP (mean, range) & $3.1(1-5)$ & $3.4(2-4)$ & $2.8(2-4)$ \\
\hline No. of experienced operators (mean, range) & $2.1(1-5)$ & $2.6(1-3)$ & $1.7(1-3)$ \\
\hline Dedicated nurses, n (\%) & $15(79)$ & $7(77)$ & $8(80)$ \\
\hline \multicolumn{4}{|l|}{ ERCP setting } \\
\hline Endoscopy room, n (\%) & $12(63)$ & $6(67)$ & $6(60)$ \\
\hline Radiology room, n (\%) & $6(32)$ & $3(33)$ & $3(30)$ \\
\hline Variable, depending on the time of the day and the patient condition, $\mathrm{n}(\%)$ & $1(5)$ & $0(0)$ & $1(10)$ \\
\hline \multicolumn{4}{|l|}{ Sex, n (\%) } \\
\hline Female & $13(21.0)$ & $10(27.0)$ & $4(16.0)$ \\
\hline Male & $49(79.0)$ & $27(73.0)$ & $21(84.0)$ \\
\hline \multicolumn{4}{|l|}{ Age group, $\mathrm{n}(\%)$} \\
\hline $29-44$ & $28(45.2)$ & $17(46.0)$ & $11(44.0)$ \\
\hline $45-54$ & $14(22.6)$ & $10(27.0)$ & $4(16.0)$ \\
\hline 55 or older & $20(32.3)$ & $10(27.0)$ & $10(40.0)$ \\
\hline \multicolumn{4}{|l|}{ Educational background, n (\%) } \\
\hline Gastroenterologist & $45(73.0)$ & $28(75.6)$ & $17(68.0)$ \\
\hline Surgeon & $14(23.0)$ & $7(19.0)$ & $7(28.0)$ \\
\hline Gastroenterologist/surgeon & $2(3.0)$ & $1(2.7)$ & $1(4.0)$ \\
\hline Gastroenterologist/Internal Medicine specialist & $1(2.0)$ & $0(0.0)$ & $1(4.0)$ \\
\hline \multicolumn{4}{|l|}{ Competence level } \\
\hline Performs ERCP independently, n (\%) & $41(66.1)$ & $25(67.6)$ & $16(64.0)$ \\
\hline Trainee, n (\%) & $21(33.9)$ & $12(42.4)$ & $9(36.0)$ \\
\hline
\end{tabular}






Fig. 1 Sedation practice.

\section{Quality monitoring}

Complication rates and cannulation success were monitored through a dedicated database that collected daily routine practice in 13 of $19(68.4 \%)$ centers.

\section{Expertise and training in experienced operators}

The majority of experienced ERCPists (32 of 41, 78\%) had performed $>1000$ ERCPs throughout their career. Fourteen of 41 operators (34\%) performed > 200 ERCP/year and were concentrated in high-volume centers ( $93 \%$ vs $7 \%$ working in mediumvolume centers). Eleven of 41 (26.8\%) experienced operators received their training during their residency/fellowship, 13 of $41(31.7 \%)$ afterwards. Before being authorized to perform ERCP independently, operators who performed 50 to 100,100 to 200 and > 200 ERCPs with the presence of a supervisor were respectively 11 of 41 (26.8\%), eight of 41 (19.5\%) and 11 of 41
(26.8\%). Four operators (9.8\%) started performing ERCP on their own, without previous supervision. The most common concern about training - mentioned by $53.6 \%$ (22 of 41 ) of experienced endoscopists - was the lack of structured training programs.

\section{Training in trainee operators}

The median time spent in ERCP training was 1.0 year (IQR, 1.03.0). Fifteen of 21 trainees (71.4\%) had performed $\leq 50$ ERCPs, three of 21 (14.3\%) had performed 101 to 200 ERCPs and three of 21 (14.3\%) had already performed 201 to 300 ERCPs. Four trainees (19\%) were training in ERCP during their residency/fellowship. Eleven of 21 trainees (52.4\%) complained about the lack of structured training programs or, if available, about their low quality. - Table 1 summarizes the data about the operator profiles; additional information about experience and training of the operators is provided as supplementary material.

Data from a total of 766 ERCP procedures performed in 19 Italian centers were recorded over a time window of 1 to 3 months. Five hundred and eighty-nine ERCPs were performed in the high-volume centers (77\%) and 177 (23\%) in the medium-volume centers. The number of procedures in each center ranged from 26 to 98 in the high-volume centers (median 62), and from nine to 30 in the medium-volume centers (median 19). Table 2 lists the indications for ERCP. There was no statistically significant difference in the grade of difficulty of ERCPs between high-volume and medium-volume centers ( $P=$ 0.796).

- Table 2 Indications for ERCP

\begin{tabular}{|c|c|c|c|}
\hline & Total $(n=766)$ & $\begin{array}{l}\text { High-volume centers } \\
(n=589)\end{array}$ & $\begin{array}{l}\text { Medium-volume } \\
\text { centers }(n=177)\end{array}$ \\
\hline Indication for ERCP & n (\%) & $n(\%)$ & n (\%) \\
\hline Biliary stones & $352(46.0)$ & $250(42.4)$ & $102(57.6)$ \\
\hline Stenting for tumors & $157(20.5)$ & $134(22.8)$ & $23(13.0)$ \\
\hline Removal/replacement of biliary stent & $106(13.8)$ & $92(15.6)$ & $14(7.9)$ \\
\hline Acute pancreatitis & $30(3.9)$ & $12(2.0)$ & $18(10.2)$ \\
\hline Recurrent pancreatitis & $13(1.7)$ & $9(1.5)$ & $4(2.2)$ \\
\hline Chronic pancreatitis & $19(2.5)$ & $17(2.9)$ & $2(1.1)$ \\
\hline Post-surgery biliary stricture & $25(3.3)$ & $20(3.4)$ & $5(2.8)$ \\
\hline Biliary leak & $23(3.0)$ & $22(3.7)$ & $1(0.6)$ \\
\hline $\begin{array}{l}\text { Diagnostic ERCP (altered laboratory testing and/or no definite } \\
\text { diagnosis on imaging and/or ERCP for tissue acquisition) }\end{array}$ & $27(3.5)$ & $21(3.6)$ & $6(3.4)$ \\
\hline Ampullary neoplasia & $9(1.2)$ & $8(1.4)$ & $1(0.6)$ \\
\hline Other (SOD, Mirizzi’s syndrome, hemobilia, benign stricture) & $5(0.6)$ & $4(0.7)$ & $1(0.6)$ \\
\hline
\end{tabular}


- Table 3 Procedures and technical success rate.

\begin{tabular}{|l|l|}
\hline Procedure & $\mathbf{n}(\%)$ \\
\hline $\begin{array}{l}\text { Successful deep cannulation of CBD in native } \\
\text { papilla }\end{array}$ & $444 / 467(95.1 \%)$ \\
\hline $\begin{array}{l}\text { Difficult cannulation } \\
\text { Double guidewire technique } \\
\text { Precut }\end{array}$ & $94 / 444(21.2 \%)$ \\
\hline Transpancreatic sphincterotomy & 26 \\
\hline Sphincterotomy & 56 \\
\hline Stone extraction<1 cm & 12 \\
\hline Success & $462 / 766(60.3 \%)$ \\
\hline Stone extraction>1 cm & $298 / 766(38.9 \%)$ \\
\hline Success & $289 / 298(97.0 \%)$ \\
\hline Partial success & $132 / 766(17.2 \%)$ \\
\hline Main pancreatic duct (MPD) cannulation & $115 / 132(87.1 \%)$ \\
\hline Unintentional MPD cannulation & $11 / 132(8.3 \%)$ \\
\hline Distal biliary obstruction stenting & $142 / 766(18.5 \%)$ \\
\hline Success & $107 / 766(14.0 \%)$ \\
\hline Hilar obstruction stenting & $264 / 766(34.5 \%)$ \\
\hline Success & $262 / 264(99.2 \%)$ \\
\hline Prophylactic pancreatic stenting & $57 / 766(7.4 \%)$ \\
\hline Papillectomy & $55 / 57(96.5 \%)$ \\
\hline Treatment of primary sclerosing cholangitis & $4 / 766(0.5 \%)$ \\
\hline Failed procedure & $53 / 766(5.6 \%)$ \\
\hline CBD, common bile duct; MPD, main pancreatic duct. & \\
\hline & $6 / 766(0.8 \%)$ \\
\hline
\end{tabular}

\section{Technical success}

A total of 713 of 766 procedures $(93.1 \%$ ) succeeded ( $\triangleright$ Table 3). A successful deep cannulation of the common bile duct in patients with a native papilla with a biliary indication for ERCP was achieved in $95.1 \%$ of cases (444 of 467). Difficult biliary cannulation occurred in 94 of 444 patients (21.2\%) and was managed by precut and/or double-guidewire and/or transpancreatic sphincterothomy technique.

Extraction of bile stones $<10 \mathrm{~mm}$ was successful in $97.0 \%$ of procedures (289/298), while complete successful extraction of stones $>10 \mathrm{~mm}$ was achieved in 115 of 132 patients (87.1\%).

Biliary stenting was successful in $99.2 \%$ of cases (262 of 264) with distal obstruction and $96.5 \%$ of patients (55 of 57) with a hilar stenosis.

\section{Operator experience and success in deep cannulation}

In our study population, we reviewed the association between the lifetime/annual workload of experienced operators and the success in deep cannulation. This analysis included cases involving patients with a native papilla. The analyzed variable of lifetime ERCP volume showed no significant differences between operators who performed $>1000$ ERCPs and those who performed < 1000 ERCPs (OR, 1.93; $95 \% \mathrm{Cl}: 0.85-4.39$; $P=0.114$ ). Similarly, the annual ERCP workload variable, defined as more or less than 200 performed ERCPs per year, was not statistically significant (OR 1.05; $95 \% \mathrm{Cl} 0.59-1.89 ; P=0.862$ ).

\section{Adverse events (AEs)}

Sixty-five ERCP-related AEs occurred in 61 patients, corresponding to a by-patient $A E$ rate of $8.0 \%$ (61 of 766, $95 \%$ Cl:6.0-10.0\%). The PEP rate was $4.0 \%$ (31 of 766 ), with a rate of $5.4 \%$ in native-papilla patients (26 of 479 ). The total bleeding rate was $2.7 \%$ (21 of 766 , nine of which were in patients on antithrombotic therapy), with a rate of $3.1 \%$ for native papillae (15 of 479). Cholangitis had a rate of $1.0 \%$ (8 of 766); there was one case of cholecystitis ( 1 of $766,0.1 \%$ ) in a patient treated with a partially covered metal stent for a distal tumor. $>$ Table 4 summarizes the AEs according to severity grade. AEs of high clinical importance (severe or fatal), occurred in only nine patients ( $1.2 \%$ ) and consisted of three perforations ( $0.4 \%)$, four bleeds $(0.5 \%)$, and two cases of acute pancreatitis $(0.3 \%$ of all the procedures). The AE rate was not statistically significantly different $(P=0.527)$ between high-volume centers $(7.6 \%, 95 \%$

- Table4 Post-ERCP adverse events according to severity.

\begin{tabular}{|c|c|c|c|c|}
\hline & Mild & Moderate & Severe or fatal & All AEs \\
\hline Pancreatitis n (\%) & $23(3)$ & $6(0.8)$ & $2(0.3)$ & $31(4.0)$ \\
\hline Bleeding n (\%) & $9(1.8)$ & $8(1.0)$ & $4(0.05)$ & $21(2.7)$ \\
\hline Cholangitis n (\%) & $1(0.1)$ & $7(0.9)$ & 0 & $8(1.0)$ \\
\hline Perforation after sphincterotomy n (\%) & 0 & 0 & $2(0.3)$ & $2(0.3)$ \\
\hline Duodenal perforation $\mathrm{n}(\%)$ & 0 & 0 & $1(0.1)$ & $1(0.1)$ \\
\hline Cardiorespiratory n (\%) & $1(0.1)$ & 0 & 0 & $1(0.1)$ \\
\hline Other $A E s^{1} n(\%)$ & $1(0.1)$ & 0 & 0 & $1(0.1)$ \\
\hline Overall ${ }^{2} \mathrm{n}(\%)$ & $35(4.6)$ & $(2.7)$ & $9(1.8)$ & $65(8.5)$ \\
\hline \multicolumn{5}{|c|}{$\begin{array}{l}\text { ERCP, endoscopic retrograde cholangiopancreatography; AE, adverse event. } \\
{ }^{1} \text { Cholecystitis } \\
2>1 \text { AE occurred in } 4 \text { patients. }\end{array}$} \\
\hline
\end{tabular}


- Table 5 Multivariable logistic regression analysis. ${ }^{1}$

\begin{tabular}{|c|c|c|c|}
\hline \multirow[b]{2}{*}{ Variable } & \multicolumn{3}{|c|}{ Clustered multivariable regression analysis } \\
\hline & ORs & $95 \% \mathrm{Cls}$ & Pvalue \\
\hline \multicolumn{4}{|c|}{ Indication: recurrent/acute/chronic pancreatitis, reference No } \\
\hline Yes & 2.57 & $1.54-3.31$ & 0.003 \\
\hline \multicolumn{4}{|c|}{ ERCP difficulty grade, reference 1} \\
\hline$>1$ & 1.57 & $0.83-2.96$ & 0.166 \\
\hline \multicolumn{4}{|c|}{ Difficult cannulation, reference No } \\
\hline Yes & 2.09 & $1.09-3.86$ & 0.033 \\
\hline \multicolumn{4}{|c|}{ ASA Classification (reference group, I) } \\
\hline II & 1.74 & $0.78-3.97$ & 0.185 \\
\hline III/IV & 1.61 & $0.70-3.78$ & 0.261 \\
\hline \multicolumn{4}{|c|}{ Success in deep cannulation VB, reference Yes } \\
\hline No & 3.47 & $1.25-9.62$ & 0.017 \\
\hline \multicolumn{4}{|c|}{ Conscious sedation } \\
\hline Deep & 2.66 & $0.44-16.07$ & 0.285 \\
\hline \multicolumn{4}{|c|}{ Prior history of ERCP, reference Yes } \\
\hline No & 1.63 & $0.61-4.37$ & 0.334 \\
\hline
\end{tabular}

Cl:5.6\%-10.1\%) and medium-volume centers (9.0\%, $95 \%$ $\mathrm{Cl}: 5.3-14.3 \%)$

Results from the clustered univariate regression analysis showed significant and positive association with post-ERCP AEs for patients undergoing examination for acute/recurrent/ chronic pancreatitis (ORs, 2.60; 95\% Cl:1.62-4.16), with difficult cannulation (ORs, 2.16; $95 \% \mathrm{Cl}: 1.08-4.34$ ) and failure in deep cannulation (ORs, 3.16; $95 \% \mathrm{Cl}: 1.42-7.08$ ). There was only a sub-threshold association of post-ERCP AEs with ASA classification $(P=0.062)$ and with the grade of ERCP difficulty $(P=0.089)$. A table containing the results of the univariate analysis for post-ERCP AEs is available as supplementary material.

In the clustered multivariable regression analysis ( $>$ Table 5), recurrent/acute/chronic pancreatitis $(P=0.003)$, difficult cannulation $(P=0.033)$ and failure in deep cannulation $(P=0.017)$ remained significantly associated with post-ERCP complications. There was no evidence of multicollinearity in the multivariable logistic regression analysis: all VIFs were less than 2.0.

\section{Outcomes data}

One month after the procedure, $90.5 \%$ of patients (693 of 766) were symptom-free. ERCP was repeated in 47 of 766 patients (6.1\%). ERCP was repeated for the management of sequelae of the procedure - failure of deep cannulation, incomplete stone extraction or management of complications - in 35 of 766 patients $(4.6 \%)$ and for the treatment of recurrent disease in 12 of 766 patients ( $1.6 \%$ ). Only three of 766 patients ( $0.4 \%$ ) died as a consequence of a severe post-ERCP AEs (two severe bleeds, one severe acute pancreatitis).

\section{Discussion}

This study showed that the overall ERCP quality in the participating Italian endoscopic centers lies within the ASGE procedural indicators targets and the ESGE performance measures. A successful cannulation of native papillae was obtained in $95.1 \%$ of procedures, a successful extraction of $<10$ - $\mathrm{mm}$ stones in $97 \%$, and a successful stent placement for distal obstruction in $99.2 \%$ of the cases, exceeding the $90 \%$ success-rate cutoff defined by the ASGE guidelines for these parameters and the $95 \%$ cutoff of the ESGE parameter for stent placement in subhilar biliary obstructions $[6,8]$. The overall technical success was $92.1 \%$, higher than that reported for previously published quality registries $[9,10,13]$. Regarding post-procedural indicators, the observed post-ERCP pancreatitis rate of $4.0 \%$ (5.4\% in intact papilla) is consistent with the available literature data [5, $6,13,19]$ and lies within both the minimum standard $(<10 \%)$ and the target standard (5\%) of the ESGE parameter. In our study, we did not observe any significant difference in cannulation rate between operators with a lifetime experience of $>1000$ ERCPs compared to $<1000$ or between operators performing more or less than 200 ERCPs per year.

Two deviations from the ASGE post-procedural indicators targets were observed. The first is a perforation rate of $0.4 \%$, equal to that of the British Society of Gastroenterology (BSG) 
audit for ERCP [9] and lower than the $0.6 \%$ reported rate in an Austrian survey that included $>13,500$ procedures [13], but higher than the $<0.2 \%$ target defined by ASGE. Published data globally report an expected incidence of $<0.6 \%$ [5] and the level of the ASGE recommendation is very weak (2C); also, perforation is a rare complication, so our sample size is too little to draw accurate conclusions.

The second deviation is a bleeding rate of $2.7 \%$, clinically significant in $1.6 \%$ of the cases compared to the recommended $\leq 1 \%$ rate. Unfortunately, the data in this study were not specifically collected to include a recording of the type and dosing/ timing of resumption of antithrombotic agents peri-procedurally administered to patients, which might partially or totally explain the observed deviation. Strikingly, however, only one of the four patients who experienced a severe hemorrhage was on antithrombothic therapy and none of them had a difficult cannulation. The previously cited Austrian survey reported an even higher total bleeding rate of 3.6\% [13] and a very recent Italian regional study also reported a rate of $2.9 \%$ [14].

The incidence of cholangitis was $1.0 \%$, which is equal to or lower than that of previously published data $[5,8,13]$.

We did not observe any significant difference in AE rates between medium- and high-volume centers. A difficulty grade II/ III was associated with a higher rate of complications, as previously reported [20], in the univariable analysis, although the significance was not confirmed by the multivariable analysis.

Nevertheless, after analyzing the baseline characteristics of the included centers, the emergence of some critical issues is evident. Although gastrointestinal endoscopy has evolved with advanced technology and procedures in the last two decades, sedation practice in the Italian endoscopy centers has not evolved at the same pace. The routine use of conscious sedation in over one-third of centers (37\%) and the lack of an anesthesiologist attendance in $28 \%$ of centers, unless the patient is in critical conditions, is an element of concern. Medium-volume centers, in particular, lack the appropriate sedation practices for such a technically demanding procedure.

Furthermore, some centers still do not administer routine prophylaxis for post-ERCP pancreatitis and an unexpectedly high number of centers (almost $40 \%$ ) did not provide an EUS service.

The most expected critical issue, however, was training to perform ERCP. Although ERCP is a technically demanding procedure, teaching programs are still lacking and this was confirmed by the questionnaire answers of the operators regarding their education. Most of the operators complained about the lack of dedicated ERCP training programs. ERCP continues to be performed by operators with varying degrees of training, experience, and procedures-per-year volumes. Only $26.8 \%$ of the experienced operators met the previously defined benchmark for ERCP competence acquisition of 200 tutored procedures before gaining autonomy $[21,22]$. Strikingly, some operators are purely self-taught. This is even more relevant as that minimum number of procedures has been debated [23]. A very recent prospective multicenter study on learning curves in advanced endoscopy trainees showed that the acquisition of competence is more probably achieved after 250 to 300 ERCPs [24] and teaching programs are shifting towards competency-based systems instead of minimum-number systems [25].

In addition, performance measures are not routinely recorded in $>30 \%$ of centers. As a result, only a few patients to date are able to determine the performance skills and competence of the ERCP operator/center before their procedures are performed.

This study has some limitations. First of all, only 19 centers participated, $47 \%$ of which performed a high number of ERCPs per year and none of which performed $<100$ ERCPs/year. Similarly, the majority of ERCP operators had good experience (>1000 ERCPs performed during their career) and a medium to high number of ERCPs performed per year. Second, the number of procedures performed and recorded is not large enough to give statistically significant results regarding some of the analyzed variables, both on a per-center and a global basis. Finally, although it was not one of the main aims of this study, the effective contribution of the ERCP trainees to each procedure was not recorded, so it is impossible to draw conclusions about their level of competence or the quality of their teaching programs. The REQUEST study did not provide new definitive indications to establish qualitative and quantitative parameters to set the minimum level of skill to proficiently perform ERCP.

\section{Conclusions}

In conclusion, our study showed that ERCP is performed meeting good quality standards and by competent operators in the participating Italian centers. The study also revealed that standardization of ERCP-related practices (i.e. sedation strategies, training programs) remains an unmet need. Training and maintaining competence, in particular, are keys to success with ERCP. As previous studies have already shown, a yearly number of $<50$ ERCPs is significantly associated with higher failure and $A E$ rates $[10,12]$. According to the average ERCP requirements in the population $(1: 1000)$ [26], we believe that the real need for ERCP operators and annual workload for each operator should be constantly evaluated to improve ERCP quality. The minimum annual workload for operators (targeting 75-100 ERCPs per year) and for centers (150-200 ERCP per year) should be pursued and traceability of procedures with collection of ERCP quality data should be ensured. Furthermore, requirements for learning centers and tutors should be established to guarantee an accurate and structured educational program.

\section{Competing interests}

The authors declare that they have no conflict of interest.

\section{References}

[1] Christensen M, Matzen P, Schulze S et al. Complications of ERCP: a prospective study. Gastrointest Endosc 2004; 60: 721-731

[2] Salminen P, Laine S, Gullichsen R. Severe and fatal complications after ERCP: analysis of 2555 procedures in a single experienced center. Surg Endosc 2008; 22: 1965-1970 
[3] Colton JB, Curran CC. Quality indicators, including complications, of ERCP in a community setting: a prospective study. Gastrointest Endosc 2009; 70: 457-467

[4] Cotton PB, Garrow DA, Gallagher ] et al. Risk factors for complications after ERCP: a multivariate analysis of 11,497 procedures over 12 years. Gastrointest Endosc 2009; 70: 80-88

[5] Chandrasekhara V, Khashab MA. ASGE Standards of Practice Committee. et al. Adverse events associated with ERCP. Gastrointest Endosc 2017; 85: 32-47

[6] Baron TH, Petersen BT, Mergener K et al. Quality indicators for endoscopic retrograde cholangiopancreatography. Gastrointest Endosc 2006; 63: S29-S34

[7] Adler DG, Lieb JG 2nd, Cohen J et al. Quality indicators for ERCP. Gastrointest Endosc 2015; 81: 54-66

[8] Domagk D, Oppong KW, Aabakken L et al. Performance measures for endoscopic retrograde cholangiopancreatography and endoscopic ultrasound: A European Society of Gastrointestinal Endoscopy (ESGE) Quality Improvement Initiative. United European Gastroenterol ] 2018; 6: 1448-1460

[9] Williams E], Taylor S, Fairclough P et al. Are we meeting the standards set for endoscopy? Results of a large-scale prospective survey of endoscopic retrograde cholangio-pancreatography practice Gut 2007; 56: 821-829

[10] Ekkelenkamp VE, de Man RA, Ter Borg F. Prospective evaluation of ERCP performance: results of a nationwide quality registry. Endoscopy 2015; 47: 503-507

[11] Coté GA, Keswani RN, Jackson T et al. Individual and practice differences among physicians who perform ERCP at varying frequency: a national survey. Gastrointest Endosc 2011; 74: 65-73.e12

[12] Kapral C, Duller C, Wewalka F et al. Case volume and outcome of endoscopic retrograde cholangiopancreatography: results of a nationwide Austrian benchmarking project. Endoscopy 2008; 40: 625630

[13] Kapral C, Mühlberger A, Wewalka F et al. Quality assessment of endoscopic retrograde cholangiopancreatography: results of a running nationwide Austrian benchmarking project after 5 years of implementation. Eur J Gastroenterol Hepatol 2012; 24: 1447-1454

[14] Mariani A, Segato S, Anderloni A et al. Prospective evaluation of ERCP performance in an Italian regional database study. Dig Liver Dis 2019; 51: $978-984$
[15] Keswani RN, Qumseya B], O'Dwyer LC et al. Association between endoscopist and center endoscopic retrograde cholangiopancreatography volume with procedure success and adverse outcomes: a systematic review and meta-analysis. Clin Gastroenterol Hepatol 2017; 15: $1866-1875$

[16] Varadarajulu S, Kilgore ML, Wilcox CM et al. Relationship among hospital ERCP volume, length of stay, and technical outcomes. Gastrointest Endosc 2006; 64: 338-347

[17] Cotton PB. Income and outcome metrics for the objective evaluation of ERCP and alternative methods. Gastrointest Endosc 2002; 56: S283-S290

[18] Cotton PB, Lehman G, Vennes ] et al. Endoscopic sphincterotomy complications and their management: an attempt at consensus. Gastrointest Endosc 1991; 37: 383-393

[19] Kochar B, Akshintala VS, Afghani E et al. Incidence, severity, and mortality of post-ERCP pancreatitis: a systematic review by using randomized, controlled trials. Gastrointest Endosc 2015; 81: 143-149

[20] Torun S, Parlak E, Yıldız H et al. Assessment of the endoscopic retrograde cholangiopancreatography grading system: A prospective study from a tertiary care center. Turk J Gastroenterol 2016; 27: 187 191

[21] Jowell PS, Baillie J, Branch MS et al. Quantitative assessment of procedural competence. A prospective study of training in endoscopic retrograde cholangiopancreatography. Ann Intern Med 1996; 125: 983-989

[22] Faulx AL, Lightdale JR et al. ASGE Standards of Practice Committee. Guidelines for privileging, credentialing, and proctoring to perform GI endoscopy. Gastrointest Endosc 2017; 85: 273-281

[23] Cotton PB. ASGE guidelines for ERCP competence. Gastrointest Endosc 2017; 86: 1190

[24] Wani S, Han S, Simon V et al. Setting minimum standards for training in EUS and ERCP: results from a prospective multicenter study evaluating learning curves and competence among advanced endoscopy trainees. Gastrointest Endosc 2019; 89: 1160-1168.e9

[25] Wani S, Keswani RN, Han S et al. Competence in endoscopic ultrasound and endoscopic retrograde cholangiopancreatography, from training through independent practice. Gastroenterology 2018; 155 : 1483-1494.e7

[26] Jovanovic I, Mönkemüller K. Quality in endoscopy training-the endoscopic retrograde cholangiopancreatography case. Ann Transl Med 2018; 6: 264 